\title{
The essential role of autophagy with oncogenes, carcinogenesis, and immune function
}

\author{
Lawrence M Agius* \\ Department of Pathology, Mater Dei Hospital, University of Malta Medical School, Malta
}

\begin{abstract}
Dynamics of dually functioning autophagic core machinery are central to the operative response of cells exposed to a potentially tumor-enhancing micro-environment that progresses further towards an initially established cancerous lesion. In such setting, further evolutionary pathways confirm the canonical role of autophagy as a primarily tumor suppressor. The interactions of the autophagy core machinery with oncogenes and tumor suppressors such as p53 are indicators of index phenomena of autophagy in the later potential establishment of a tumor focus. Hence, autophagy is both directly and indirectly influenced by systems of proinflammatory cytokine/chemokine action. In terms of ongoing dynamics of tumorigenesis, autophagy may fulfill the roles of cellular forms of immunity to intracellular events such as invasive bacteria and stress phenomena of otherwise ongoing carcinogenic nature. Selective autophagy is essential in the turnover of multiple cellular substrates, exerting crosstalk with phagocytosis in inflammation and immunity. The autophagy core machinery is highly conserved evolutionarily. In tumorigenesis, $\mathrm{NF}-\kappa \mathrm{B}$ (Nuclear Factor kappa-B) signaling, Nrf2 transcription factor NF-E2-related factor 2) and apoptosis pathways are activated in cells with defective autophagy.
\end{abstract}

Autophagy deficient cells accumulate p62 protein (an adaptor autophagy protein) and this appears important in NF- $\mathrm{KB}$ regulation and inflammation. Autophagy deficient cells are prone to necrosis or apoptosis, and thus may enhance inflammation.

\section{Introduction}

The concept of dynamic autophagy offers an attractive constitutive mechanism for tumor suppression in the initial stages of tumorigenesis. Cell death induced by the small molecule compound YM155 (the first "Survivin suppressant") critically depends on crosstalk between DNA damage and a DNA damage response in p53-proficient breast cancer cells [1]. In such setting, the turnover of cytosol molecules and of whole organelles such as portions of the endoplasmic reticulum and integral but abnormal mitochondria convey the mechanistic scope of turnover inherent in maintenance of normal cell functionalities. Epigallocatechin gallate (EGCG) inhibits cell viability of oral cancer resistant to cisplatin and induces cell apoptosis and autophagy [2].

\section{Cell transformation}

The integral phenomenon of cell transformation in carcinogenesis arises within the constitutional parameters of change as related to cell stress and particularly in relation to genetic damage as that arising due to effective excess of oxidation free radical production.

Raised levels of p62 lead to activated NRF2 and mTORC1 (mammalian target of rapamycin complex 1), induced c-Myc protooncogene and hepatocarcinoma [3]. The oxidative injury to cells and also other forms of cell stress such as the accumulation of protein aggregates or of mis-folded molecular species is intermediary molecular representation of reactive cell response to a host of other putative stress pathways.

These in turn may be suggestive of certain aspects of the inherent nature of the malignant transformation processes themselves. The oncogene lysosome-associated protein transmembrane- $4 \beta$ could be a poor tumor prognostic marker [4], promoting autophagy and inhibiting apoptosis.

\section{Essential dynamics}

Dynamics of cellular constituent processes allow for the emergence of stress responses that may potentially delineate a series of pathway abnormalities as exemplified by excess autophagy activities. Beclin 1 (homologue of yeast autophagy gene protein $8, \mathrm{Bcl}-2$ interacting protein) effects a significant role in inducing autophagy during carcinogenesis and may constitute an important target in lung carcinoma [5]. Beclin 1 interacts with canonical NF- $\mathrm{KB}$ and thus appears to coordinate response to stress.

The unfolded protein response may activate autophagy and is in turn induced by cytokines such as IFN- $\gamma$ (Interferon-gamma), IL-1 (Interleukin-1), TGF- $\beta$ (Transforming Growth Factor gamma) and TNF-a (Tumor Necrosis Factor-alpha).

The determined constitutive attributes of parametric modulation is better exemplified by the appearance of autophagic vesicles as doublemembrane organelles in their own right and furthermore would include the fusion of transported vesicles with the lysosomal single membrane. The transportation and release of contained debris within the autophagic vesicles is determined by various receptors that allow for the release of abnormal organelles such as mitochondria. Autophagy dysfunction is associated with inflammatory bowel disease and colorectal cancer [6]. Megestrol acetate on the other hand diminishes skeletal and heart muscle wasting in cancer cachexia, through a marked downregulation of autophagy [7].

Correspondence to: Lawrence M Agius, 27 "BALLARAT", Guzeppe Caruana Street, Tal-Virtu, Rabat, RBT09, Malta, Tel: 356-21451752; E-mail: lawrence.agius@um.edu.mt

Received: May 27, 2016; Accepted: June 13, 2016; Published: June 16, 2016 


\section{Tumor suppression}

Determinant tumor suppressive function of autophagy in initial steps of a multi-step formulation of malignant transformation is hence a directed and specifically active series of pathway dynamics that cooperates with such modulators as the mTOR and p53-ARF (tumor suppressor protein, ADP-ribosylation factor) mechanisms. MicroRNAs may act as tumor suppressors or oncogenes, within the context of autophagy as exemplified by micro-RNAs MIR376A and MIR476B [8].

It is interesting to view oncogene and suppressor gene effects that inherently cooperate in decreasing or increasing respectively the autophagic activities of cells in tumorigenesis. Notch3 appears to be crucial in increasing stem-like properties in non-small cell lung carcinoma and may be related to activated autophagy [9].

What emerges from such considerations is the central series of roles that determine the importance of interactivities between molecules and cells found in the tumor micro-environment. Beclin 1 in particular binds to Bcl-2 on the ER and is released through phosphorylation by c-Jun $\mathrm{N}$-terminal kinase 1 under nutrient starvation. UVRAG interacts with Beclin 1 and is commonly monoallelically deleted in cancers such as colon and gastric carcinoma.

Ubiquitination of connexins is implicated in autophagy-mediated degradation of $\mathrm{Cx} 43$ via the proto-oncogenic E3 ubiquitin ligase NEDD4, with loss of intercellular communication via gap junctions in response to oxidative stress and in carcinogenesis [10].

The distributional dynamics of the cancer-associated fibroblasts (CAFs) with endothelial cells is particularly relevant to the initial stages and progression of the angiogenic switch that allows further tumor growth. CAFs promote growth and spread of neoplastic cells via autophagy and aerobic glycolysis to feed anabolic tumor cells. Growth factors and cytokine/chemokine biology appear to operate within such micro-environmental conditions that actively and also constitutively permit the emergence of critical alterations in pathways that impact on the autophagic machinery of the target cells. M2 macrophage polarization promotes vascular permeability and carcinogenesis in urethane carcinogenesis with implications in abnormal angiogenesis [11].

\section{Autophagy enhancement}

The malignant transformation process is a crucial characterization of the paramount dynamic nature of carcinogenesis whereby predominantly indirect effects of impaired autophagic activities lead to accumulation of stress stimuli within target cells. Hepatic carcinogenesis involves altered gap junctions, the proteasome/autophagy system and mitochondrial dysfunction [12].

The emergence of genetic lesions due to both intrinsic cellular and non-cell autonomous effects such as inflammation in the microenvironment of tumors allows for the abnormal mitochondria to persist within transforming cells.

\section{Cell stress}

Cell oxidative stress and such mechanisms as the reverse Warburg phenomena may provide stimuli and nutrient by-products to cells that are progressing further in the integral process of tumorigenesis. In this respect, autophagy may constitute a truly dual role in the further enhancement of tumorigenesis in a manner that permits the pathway dynamics to become dynamically established both as proliferative foci and also as infiltrating and metastasizing lesions. Cisplatin may induce apoptosis and autophagy and autophagy may inhibit apoptosis in MG63 osteosarcoma cell lines [13]. Negative expression of tuberous sclerosis 2 is implicated in a link between epigenetic regulation, the mTOR pathway, autophagy induction and carcinogenesis [14].

Inclusive parameters of dynamic turnover of portions of the cell components are related also to endoplasmic reticulum stress with the accumulation of mis-folded and aggregated protein molecules.

Autophagy is stimulated in a process of hermesis in relative reference to a series of permissive stress conditions that may stimulate autophagic activity. The beneficial biologic effects of exposure to mild levels of toxin and other forms of cell injury permit hermesis to operate and maintain basal and mildly increased levels of autophagic activity within cells in a manner that further promotes a multi-stage tumorigenesis in some such targeted cells. MAP1S (an autophagy activator), promotes biogenesis and degradation of autophagosomes, triggers degradation of lipid droplets in clear cell renal cell carcinoma, and enhances initiation and development of this tumor type [15].

\section{Pathway effects}

The mTOR/PI3K/AKT pathway operates to decrease autophagy whereas the p53/ARF pathway operates to enhance autophagy. 35 autophagy-related genes (Atg) have so far been cloned and deletion/ mutation of many of these genes is found in some cancerous lesions. In this respect mono-allelic loss of the Beclin 1 is associated with the development of various neoplasms in mouse models, including lymphoma, hepatocarcinoma and other types of tumor. In particular, the decreased autophagic activity found in mono-allelic loss of the Beclin 1 gene allows for increased sensitivity of the target cells to undergo tumorigenesis when exposed to chemical carcinogens.

DAMPs react with DAMP receptors such as TLRs and thus activate NF- $\kappa B$ and also PI3K (Phosphatidyl-inositol 3-Kinase)/AKT serine/ threonine kinases and mitogen activated protein kinases and thus contribute to inflammatory reactivity and cell death. NF- $\mathrm{kB}$ contributes to regulated apoptosis and survival of immune cells and cells with damaged DNA and in cancers. Hypoxia inducible factor regulates autophagy, apoptosis, angiogenesis and cell cycling in response to microenvironmental levels of hypoxia. The unfolded protein response may activate autophagy in a hypoxic environment via PERK (Prolinerich extensin-like receptor)-activating transcription factor 4 pathway on the ER. Huge aggregates of ubiquinated proteins are sequestered into autophagosomes by p62 and NBR1 (autophagy cargo receptor neighbor of BRCA1) autophagy receptor and thus degraded. In tumorigenesis, NF- $\mathrm{kB}$ signaling, Nrf2 and apoptosis pathways are activated in cells with defective autophagy.

\section{Tumor micro-environment}

The tumor micro-environment is a central hub for tumorigenesis and this has become increasingly recognized as further promoting such systems as cell autophagy. DNA damage involves factors in the activation of autophagy and of apoptosis and necrosis; these determine the outcome of cancer therapy with genotoxic agents [16].

The multitude of micro-environmental components that include in particular not only immune and pro-inflammatory cytokines and chemokines relates to a multi-step process of carcinogenesis that distinctively includes separate distinct phases in the initiation and also later progression of the established tumor lesion. In such dynamic context, autophagy is tumor suppressive in the initial stages of 
tumorigenesis, but later enhances progression of the once-established tumor growth phase. Notably, Kirsten rat sarcoma-driven tumors rely on macroautophagy for growth and survival, thus suggesting a potential therapeutic approach for autophagy inhibitors based on genetic stratification [17].

Mosaic mouse models of the atg (autophagy related gene) 5 and atg 7 have helped indicate the essential specificity of context-dependent tumorigenesis in such organs as the liver. In such a setting atg 5-/hepatocytes may undergo malignant transformation whereas adjacent hepatocytes with normal atg 5 complement are unaffected.

Atg5-deficiency in hepatic progenitor cells disrupts hepatic differentiation; autophagy may contribute to stemness maintenance and reduces susceptibility to carcinogenesis in hepatic progenitor cells [18].

\section{Inflammation}

nflammation invokes various transcription factors such as NF$\mathrm{\kappa B}$ that in turn helps orchestrate a whole series of targeting actions in carcinogenesis. Direct crosstalk between NF- $\kappa B$ and the canonical autophagy machinery occurs with NF- $\mathrm{kB}$ either stimulating or inhibiting autophagy. The autophagy core machinery is hence related in multi-pathway manner with the processes arising from stressinduced exposure to a multitude of potential carcinogenetic agents. Helicobacter pylori can act through toll-like receptor-4 (TLR-4) and induction of cell autophagy, and induce gastric mucosal inflammation and carcinogenesis [19].

\section{Damage-associated molecular patterns}

Damage-associated molecular patterns, including in particular high mobility group box 1 are particularly relevant to the receptivity component pathways in carcinogenesis. Also important DAMP factors such as ATP, S100 and heat shock protein (especially HSP70) may be released from necrotic or apoptotic cells and stimulate in turn proinflammatory pathways, including dendritic cells, and also immune cells such as lymphocytes.

\section{Concluding remarks}

Clearly, increased autophagy has an overall tumor suppressor role in tumorigenesis and is therefore a fundamental modulator in determining the potential establishment of a tumor focus. Transforming growth factor-beta mediates activation of autophagy and may promote growth inhibition of hepatocarcinoma cells [20]. Added to such tumor suppression is the processing of intracellular processes and secretory activities of the ER and Golgi apparatus in the handling and targeting actions of cytokines. ER stress in particular, like also nutrient deprivation, is a powerful stimulant of increased autophagy, whereby accumulating protein aggregates and damaged portions of the ER can be sequestered and degraded within lysosomes. Such process is important also in view of the relatively small proteasome in some of the targeted cells that may not effectively turnover and remove mis-folded proteins and abnormal mitochondria or peroxisomes. Progranulin is upregulated in psoriasis and inhibits inflammation by decreasing production of inflammatory factors and positively inducing autophagy through the Wnt/Beta-catenin signaling pathway [21]. Autophagy helps regulate cellular immunity and $\mathrm{T}$ cell maturation, with intracellular antigen presentation to MHC class molecules [22]. Also, within a contextual framework, autophagy may also support tumorigenesis with increased access to nutrients, inhibition of cell death and increased drug resistance [23].
The derivation of the outer membrane of autophagic vesicles as related to the plasma membrane or ER emphasizes the constitutive nature of a basal autophagic activity that characterizes the potential future response to exposure of cells to innumerable forms of stress or carcinogenic agents.

\section{Immune system/autophagy responses}
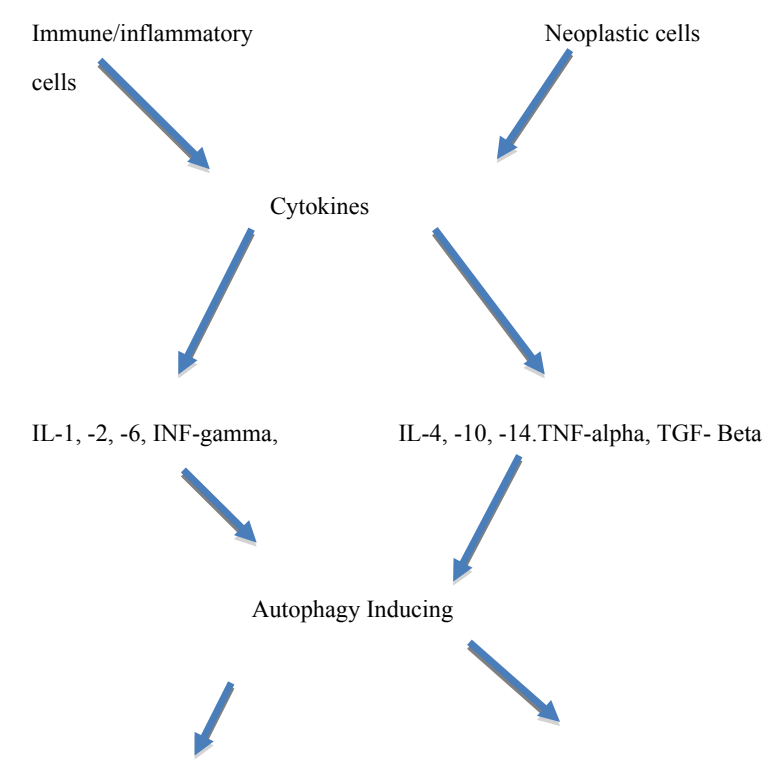

Anti-neoplastic Response

Pro-neoplastic Response

\section{References}

1. Vaquaud E, Saveno C, Loussouarn D, Engelhart L, et al. (2015) YM155 potently triggers cell death in breast cancer cells through an autophagy-NF- $\kappa \mathrm{B}$ network. Oncotarget 6: 13476-13486.[Crossref]

2. Yuan CH, Horng CT, Lee CF, Chiang NN, Tsai FJ, et al. (2016) Epigallocatechin gallate sensitizes cisplatin-resistant oral cancer CAR cell apoptosis and autophagy through stimulating AKT/STAT3 pathway and suppressing multidrug resistance 1 signaling. Environ Toxicol [Crossref]

3. Umemura A, He F, Taniguchi K, Nakagawa H, Yamachika S, et al. (2016) â€œp6, upregulated during preneoplasia, induces hepatocellular carcinogenesis by maintaining survival of stressed HCC-initiating cells. Cancer Cell [Crossref]

4. Meng Y, Wang L, Chen D, Chang Y, Zhang M, et al. (2016) LAPTM4B: an oncogene in various solid tumors and its functions. Oncogene.[Crossref]

5. Wang W, Fan H, Li X, Wu G, Zhao W, Zhang G et al. (2016) Beclin 1 promotes apoptosis and decreases invasion by upregulating the expression of ECRG4 in A549 human lung adenocarcinoma cells. Mol Med Rep.[Crossref]

6. Musolino V, Palus S, Tschimer A, Drescher C, Gliozzi M, et al. (2016) Megestrol acetate improves cardiac function in a model of cancer cachexia-induced cardiomyopathy by autophagic modulation. J Cachexia Sarcopenia Muscle [Crossref]

7. Talero E, Alcaide A, Avila-Roman J, Garcia-Maurifio S, Vendramini-Costa D, et al Expression patterns of sirtuin 1-AMPK-autophagy pathway in chronic colitis and inflammation-associated colon neoplasia in IL-10-deficient mice. Int Immunopharmacol 35: 248-256. [Crossref]

8. Tekirdag KA, Akkoc Y, Kosar A, Gozuacik D (2016) MIR376 family and cancer. Histol Histopathol.[Crossref]

9. Ma Y, Li M, Si J, Xiong Y, Lu F, et al. (2016) Blockade of Notch3 inhibits the stem-like property and is associated with LDH1A1 and CD44 via autophagy in non-small lung cancer. Int J Oncol 48: 2349-2358. [Crossref]

10. Leithe E (2016) Regulation of connexins by the ubiquitin system: implications for intercellular communication and cancer. Biochim Biophys Acta 1865: 133-146. [Crossref] 
11. Li GG, Guo ZZ, Ma XF, Cao N, Geng SN, et al. (2016) The M2 macrophages induce autophagic vascular disorder and promote mouse sensitivity to urethane-related lung carcinogenesis. Dev Comp Immunol 59: 89-98. [Crossref]

12. Tork OM, Khaleel EF, Abdelmaqsoud OM (2015) Altered cell to cell communication, autophagy and mitochondrial dysfunction in a model of hepatocellular carcinoma: potential protective effects of curcumin and stem cell therapy. Asian Pac J Cancer Prev 16: 827-829. [Crossref]

13. Zhang Z, Shao Z, Xiong L, Yang S (2015) Inhibition of autophagy enhances cisplatininduced apoptosis in the MG63 human osteosarcoma cell line. Oncol Lett 10: 29412946.[Crossref]

14. Wei FZ, Cao Z, Wang X, Wang H, Cai MY, et al. (2015) Epigenetic regulation of autophagy by the methyltransferase EZH2 through an MTOR-dependent pathway. Autophagy 11: 2309-2322.[Crossref]

15. Xu G, Jiang Y, Xiao Y, Liu XD, Yue F, et al. (2016) Fast clearance of lipid droplets through MAP1S-activated autophagy suppresses clear cell renal cell carcinomas and promotes patient survival. Oncotarget 7: 6255-6265. [Crossref]

16. Roos WP, Thomas AD, Kaina B1 (2016) DNA damage and the balance between survival and death in cancer biology. Nat Rev Cancer 16: 20-33.[Crossref]

17. Eng CH, Wang Z, Tkach D, Toral-Barza L, Ugwonali S, et al. (2016) Macroautophagy is dispensable for growth of KRAS mutant tumors and chloroquine efficacy. Proc Natl Acad Sci U S A 113: 182-187.[Crossref]

18. Xue F, Hu L, Ge R, Yang L, Liu K, et al. (2016) Autophagy-deficiency in hepatic progenitor cells leads to the defects of stemness and enhances susceptibility to neoplastic transformation. Cancer Lett 371: 38-47. [Crossref]

19. Meng W, Bai B, Sheng L, Li Y, Yue P, et al. (2015) Role of Helicobacter pylori in gastric cancer: advances and controversies. Discov Med 20: 285-293.[Crossref]

20. Korah J, Canaff L, Lebrun JJ (2016) The retinoblastoma tumor suppressor protein $(\mathrm{pRb}) / \mathrm{E} 2$ promoter binding factor 1 (E2F1) pathway as a novel mediator of TGF $\beta$ induced autophagy. J Biol Chem 291: 2043-2054

21. Tian R, Li Y, Yao X (2016) PGRN Suppresses Inflammation and Promotes Autophagy in Keratinocytes Through the Wnt/ $\tilde{\mathrm{A} Z} \hat{\mathrm{A}}^{2}$-Catenin Signaling Pathway. Inflammation. [Crossref]

22. Oral O, Yedier O, Kilic S, Gozuacik D (2016) Involvement of autophagy in T cel biology. Histol Histopathol.[Crossref]

23. Burada F, Nicoli ER, Ciurea ME, Uscatu DC, Ioana M, et al. (2015) Autophagy in colorectal cancer: An important switch from physiology to pathology. World $J$ Gastrointest Oncol 7: 271-284.[Crossref]

Copyright: (2016 Agius LM. This is an open-access article distributed under the terms of the Creative Commons Attribution License, which permits unrestricted use, distribution, and reproduction in any medium, provided the original author and source are credited. 